www.jmscr.igmpublication.org

Impact Factor 5.244

Index Copernicus Value: 83.27

ISSN (e)-2347-176x ISSN (p) 2455-0450

crossref DOI: _https://dx.doi.org/10.18535/jmscr/v4i12.34

Journal Of Medical Science And Clinical Research

\title{
A Prophylactic Phenylephrine Infusion for Preventing Hypotension during Spinal Anaesthesia for Caesarean Section and to Study its Effects on APGAR Score and Umbilical Artery Blood pH
}

\author{
Authors \\ Bilal Mohammad $^{1 *}$, Pavan Dhulkhed ${ }^{2}$, P.B. Jamale ${ }^{3}$, Tushar Munnoli ${ }^{4}$, V.K. Dhulkhed ${ }^{5}$ \\ ${ }^{1,4}$ Postgraduate Student, ${ }^{3}$ Professor, ${ }^{5}$ Professor \& HOD, Dept. of Anaesthesiology, Krishna Institute of \\ Medical Sciences, Karad, Maharashtra \\ ${ }^{2}$ Assistant Professor, Dept. of Anaesthesiology, J N Medical College, Belagavi, Karnataka \\ *Corresponding Author \\ Dr Bilal Mohammad
}

Post Graduate Student, Department of Anaesthesiology,

Krishna Institute of Medical Sciences, Karad, Maharashtra, India

Email: dr.bilal1204@gmail.com, Mobileno-9130245696

\begin{abstract}
BACKGROUND: Hypotension after spinal anaesthesia for caesarean section is a common complication. The usual approach to use vasopressors in this clinical setting is reactive rather than proactive; spinal anaesthesia induced hypotension is allowed to develop and then treated accordingly. We have, insteadstudied prophylactic phenylephrine infusion for prevention of hypotension and its effects on APGAR score and umbilical artery blood $\mathrm{pH}$.

METHOD: Nonlaboring patients scheduled for elective cesarean sections were randomly allocated into two groups of 30 each. Group I received intravenous prophylactic phenylephrine infusion at 100 mcg minute-1for 3 minutesafter spinal anaesthesia. Then each minute systolic arterial pressure(SAP) was measured and infusion stopped if SAP> $20 \%$ baseline and continued or restarted, otherwise. Intravenous phenylephrine bolus $100 \mathrm{mcg}$ was given when SAP is decreased to <20\% of baseline. Group II received ephedrine $6 \mathrm{mg}$ bolus only when SAP decreased to $<20 \%$ of base line. Afterlminute of spinal anaesthesia; Heart rate (HR), SAP were recorded every minute till the extraction of the baby. After the delivery of the baby, APGAR score at 1 and 5 minutes were noted. Umbilical artery blood pHwas analysed.

RESULTS: Prophylactic phenylephrine infusion decreased the incidence (2of 30 [6.66\%] versus 29 of $30[96.66 \%])$ of hypotension compared with control. Heart rate was significantly lower in the infusion group. Umbilical artery blood $\mathrm{pH}$ and APGAR scores were similar.

CONCLUSION: Prophylactic phenylephrine infusion is an effective and simple method of reducing the incidence of hypotension during spinal anaesthesia for cesarean delivery, with no adverse effect on neonatal outcome.

KEYWORDS: Prophylactic Phenylephrineinfusion, Spinalanaesthesia,Maternal hypotension, Cesarean section.
\end{abstract}




\section{Introduction}

In the recent decades there has been a worldwide shift in obstetric anaesthesia practice in favour of regional anaesthesia with spinal anaesthesia being the most popular among them. ${ }^{1}$ But, like any other anaesthetic technique, it is not devoid of complications, the most common being hypotension which may adversely affect both mother and fetus.

In caesarean section under spinal anaesthesia hypotension has been reported in as many as $85 \%$ of the patients. ${ }^{2}$ It can cause fetal bradycardia ${ }^{3}$ and acidosis ${ }^{4}$ and is associated with symptoms like nausea, vomiting which may interfere with surgical procedure.Measures to decrease the incidence and severity of maternal hypotension include left uterine displacement, trendelenburg position, leg compression, fluid preloading etc., but these are not complete measures ${ }^{5,6}$ and vasopressor is required to correct hypotension quickly. $^{7}$

Traditionally, ephedrine has been the vasopressor of choice in pregnant women. The use of $\alpha$ agonists has generally been avoided since the 1970s because of concerns about their potential adverse effect on uterine blood flow. However, in a quantitative, systematic review of randomized controlled trials of ephedrine versus phenylephrine for the management of hypotension during spinal anaesthesia for cesarean delivery, Lee and colleagues showed that there was no difference between ephedrine and phenylephrine in efficacy. They did find, however, that women given phenylephrine had neonates with higher umbilical cord blood $\mathrm{pH}$ values than women given ephedrine, although the risk of true fetal acidosis (umbilical $\mathrm{pH}$ value of 7.20) was similar in both groups. Because acidotic changes in the umbilical arterial $\mathrm{pH}$ are sensitive indicators of reduced uteroplacental perfusion, the authors concluded that their finding was indirect evidence that uterine blood flow may in fact be better with phenylephrine compared with ephedrine. ${ }^{8}$

The usual approach to use vasopressors in this clinical setting is reactive rather than proactive; spinal anaesthesia induced maternal hypotension is allowed to develop and then treated accordingly. Very few studies have compared prophylactic phenylephrine infusion and ephedrine boluses for treatment of hypotension following spinal anaesthesia. The aim of our study was to compare prophylactic phenylephrine infusion for maintenance of maternal blood pressure during spinal anaesthesia in caesarean section and its effects on APGAR score and umbilical artery blood $\mathrm{pH}$ and to compare it with bolus ephedrine which is given as and when required.

\section{Material and Method}

After obtaining institutional ethical committee approval, we recruited 60 ASA physical statusII women with term singleton pregnancies scheduled for elective caesarean delivery under spinal anaesthesia in this randomised prospective double blinded study. All patients gave written, informed consent. Patients with pre-existing or pregnancy induced hypertension, cardiovascular or cerebrovascular disease, known fetal abnormalities, or contraindications to spinal anesthesia were excluded. All the patients were evaluated prior to surgery and relevant investigations were done.

Patients were preloaded with ringer lactate solution, $10 \mathrm{ml} \mathrm{kg-1}$ over 15 minutes after securing intravenous line with 20 gauge cannula and continued at $10 \mathrm{ml}$ min-1. After receiving the patient inside the operation theatre, standard monitoring, including noninvasive arterial blood pressure, electrocardiography, and pulse oximetry, was attached. Fetal heart rate (FHR) was monitored by external cardiotocography until the time of surgical preparation. Patients were allowed to rest undisturbed for several minutes, during which systolic arterial pressure (SAP) was measured every 1-2 minutes. This was continued until measurements became consistent (3 successive measurements of SAP that had a difference of no more than 10\%). Baseline SAP and heart rate (HR) were taken as the mean of the two recordings. 
Two identical syringes were prepared containing either phenylephrine $100 \mathrm{mcg} \mathrm{ml}-1$ or saline. According to computer-generated randomization codes contained in sealed, sequentially numbered envelopes, an investigator or anesthesiologist not involved with patient care or assessment then selected one of the syringes to be used for intravenous (IV) infusion and the other for IV boluses. The investigator administering the solutions and the patient were thus blinded to the contents of the syringes.

Spinal anaesthesia was administered with patients in right lateral position. After skin infiltration with lidocaine, a 25 gauge Quincke spinal needle was inserted at L2-L3 or L3-L4 vertebral interspace, and hyperbaric $0.5 \%$ bupivacaine $2.0 \mathrm{ml}$ was injected intrathecally. Patients were then immediateely turned supine. SAP was measured at 1-minute intervals beginning 1 minute after spinal injection.

Immediately after the completion of intrathecal injection, infusion of study solution was started at 1 mlmin-1. Thus, patients in the infusion group had an IV infusion of phenylephrine started at 1 mlmin-1 (100 mcg min-1), whereas patients in the control group had an IV infusion of saline started at $1 \mathrm{mlmin}-1$. Infusions were administered with a syringe pump that was connected to the IV line via a three-way stopcock and were continued for a minimum of 3 minutes, after which the infusion was either stopped or continued according to a predefined protocol based on the SAP measurement each minute. After each minute measurement of SAP, the infusion was stopped if the SAP was more than baseline, and it was continued or restarted if the SAP was less than or equal to baseline. For the purposes of the study, we defined hypotension as a decrease in SAP to less than $20 \%$ of baseline. Each time there was a SAP measurement showing hypotension, patients received a $1 \mathrm{ml}$ IV bolus of study solution. Thus, patients in the infusion group received a $1 \mathrm{ml}$ (100mcg) IV bolus of phenylephrine, and patients in the control group received a $1 \mathrm{ml}(6 \mathrm{mg}) \mathrm{IV}$ bolus of ephedrine. Dosing regimens are selected on the basis of previous studies.

Any incidences of nausea or vomiting were recorded. Nausea or vomiting that was not associated with hypotension was treated with intravenous metoclopramide $10 \mathrm{mg}$, and bradycardia, defined by an $\mathrm{HR}<50 \mathrm{bpm}$ with intravenous atropine $0.6 \mathrm{mg}$. Oxygen saturation $<95 \%$ treated by $100 \%$ oxygen supplementation through Magill's circuit. Pre-anaesthetic drill and emergency intubation kept ready.

After intrathecal injection of local anaesthetic bupivacaine, the upper sensory level of anaesthesia were measured by assessing loss of pinprick discrimination. After 1 minute of sub arachnoid block (SAB), HR, SAP were recorded every minute till the extraction of the baby. The time of skin incision, uterine incision and delivery of the baby were recorded. The infusion and bolus protocol was continued until the time of delivery of the baby, after which further management was at the discretion of the attending anaesthesiologist, who was free to administer intravenous fluids and vasopressors as appropriate to replace surgical loss and maintain SAP.

After delivery, oxytocin $20 \mathrm{IU}$ in $500 \mathrm{ml}$ saline was given by slow intravenous infusion. APGAR scores were assessed at 1 and 5 minutes after delivery. Umbilical artery blood samples were taken from a double clamped segment of umbilical cord for immediate blood $\mathrm{pH}$ analysis. Using data from previous studies, to detect $50 \%$ difference in episodes of hypotension in two groups for alpha value of 0.01 and power of $90 \%$, we calculated sample size of 22 patients per group. To allow for potential dropouts, we decided to recruit 60 patients. Statistical analysis was done by descriptive statistics as mean, SD etc. Comparison of mean values of continuous parameters under study was done by applying Student's Unpaired ' $t$ ' test at 5\% and 1\% level of significance. Mann Whitney test, Chi-square and Fisher Exact test have been used to find the significance of study categorical data as appropriate. ' $\mathrm{P}$ ' value of $<0.05$ was considered to 
be statistically significant. The statistical software namely SYSTAT version 12 (made by Cranes Software's, Bangalore) was applied.

\section{Results}

All patients completed the study. Patient characteristics and surgical times were similar between groups (Table 1).The level of sensory block obtained is also comparable in both the groups and most of the patients had average blockade level of T6. Neonatal outcome was similar between the groups (Table 2).

Serial analysis of hemodynamic changes showed that SAP was significantly greater over time in the infusion group compared with the control group ( $\mathrm{P}<0.0001$; Fig. 1) and that HR was significantly slower over time in the infusion group compared with the control group ( $\mathrm{P}<0.0001$; Fig. 2).No patient required treatment with atropine.

Cumulative survival (the proportion of patients who did not become hypotensive) over time until delivery was greater in the infusion group compared with the control group ( $\mathrm{P}<0.0001 ;$ ). Overall, $2(6.66 \%)$ of 30 patients in the infusion group had one episode of hypotension each, compared with $29(96.66 \%)$ of 30 in the control group ( $\mathrm{P}<0.0001)$, and the number of episodes of hypotension was smaller in the infusion group (2 episodes) compared with the control group (54 episodes; $\mathrm{P}<0.0001$; Table 3). The two patients in the infusion group had only a single episode of hypotension each. In comparison, all of the 29 patients in the control group who had hypotension had $>1$ episode, and half had $>2$ episodes. The minimum SAP recorded was lower in the control group compared with the infusion group.

In the first few minutes after intrathecal injection, SAP increased slightly more than baseline in some patients in the infusion group, whereas SAP decreased in all patients in the control group (Fig. 1). The incidence of reactive hypertension was more frequent in the infusion group compared with the control group, but no patient complained of symptoms. The maximum SAP recorded was greater in the infusion group compared with the control group (Table 3). There were no incidences of complications such as nausea and vomiting after induction of SAB in both the groups.

Table 1: Comparison of basic parameters between two Groups.

\begin{tabular}{|c|c|c|c|}
\hline \multirow[t]{2}{*}{ Parameters } & $\begin{array}{l}\text { Group I } \\
(n=30)\end{array}$ & $\begin{array}{l}\text { Group II } \\
(\mathrm{n}=30)\end{array}$ & \multirow[t]{2}{*}{ 'p' value } \\
\hline & Mean \pm SD & Mean \pm SD & \\
\hline Age in years & $24.97 \pm 7.97$ & $25.27 \pm 28.02$ & 0.397 \\
\hline Weight in Kgs. & $65.77 \pm 12.86$ & $65.9 \pm 13.99$ & 0.328 \\
\hline Height in Cms. & $157.97 \pm 32.43$ & $155.33 \pm 29.31$ & 0.463 \\
\hline${ }^{\#}$ SID Time in Sec. & $530.00 \pm 60.17$ & $528.00 \pm 57.68$ & 0.046 \\
\hline${ }^{\$}$ UID Time in Sec. & $61 \pm 7.24$ & $60 \pm 5.16$ & 0.097 \\
\hline
\end{tabular}

Table 2: Neonatal Outcome

\begin{tabular}{|c|c|c|c|}
\hline \multirow{2}{*}{ Parameters } & $\begin{array}{c}\text { Group I } \\
(\mathrm{n}=30)\end{array}$ & $\begin{array}{c}\text { Group II } \\
(\mathrm{n}=30)\end{array}$ & Mean \pm SD \\
\cline { 2 - 4 } & Mean \pm SD & $\mathrm{p}$ ' value \\
\hline APGAR SC-1 minute & $8.83 \pm 0.79$ & $9.10 \pm 0.66$ & 0.097 \\
\hline APGAR SC 5-minute & $9.67 \pm 0.47$ & $9.83 \pm 0.38$ & 0.047 \\
\hline ^UAB pH & $7.17 \pm 0.03$ & $7.17 \pm 0.03$ & $\mathrm{p}>0.05$ \\
\hline
\end{tabular}

$\wedge$ Umbilical Artery Blood pH. 
Table 3: Details of Hypotension Episodes

\begin{tabular}{|c|c|c|}
\hline Incidence of Hypotension & Group I $(n=30)$ & Group II $(n=30)$ \\
\hline Absent & $28(93.33)$ & $1(3.33 \%)$ \\
\hline Present & $2(6.66)$ & $29(96.66)^{*}$ \\
\hline Episodes & 2 & $54 *$ \\
\hline Minimum recorded ${ }^{\mathfrak{I}} \mathrm{SAP}(\mathrm{mmHg})$ & 96 & $82 *$ \\
\hline Maximum recorded ${ }^{\mathfrak{f}} \mathrm{SAP}(\mathrm{mmHg})$ & 142 & $118^{*}$ \\
\hline
\end{tabular}

*Significant difference, $\mathrm{p}<0.001, £$ Systolic Arterial Pressure.

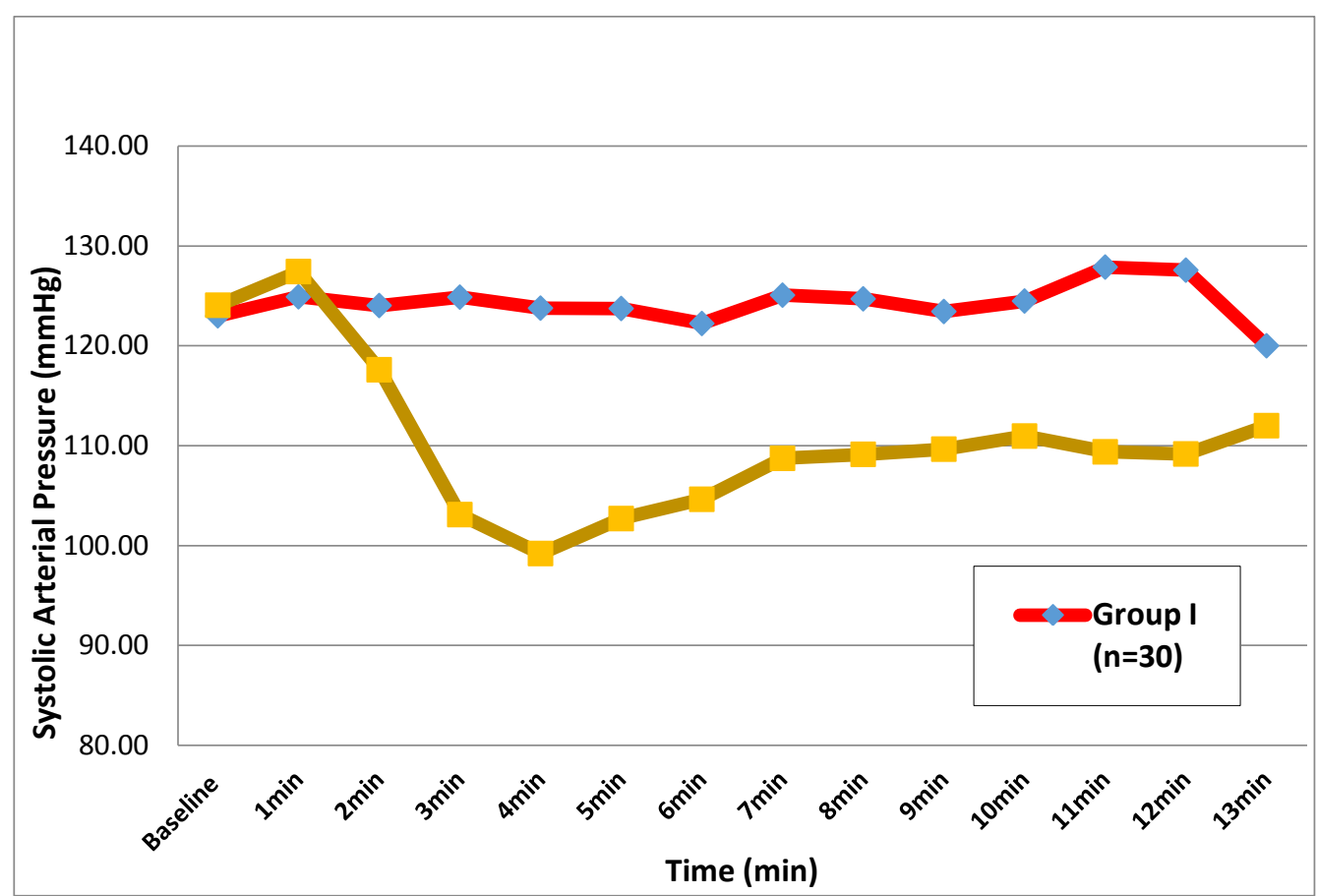

Fig 1: Comparison of Systolic Arterial Pressure among two groups

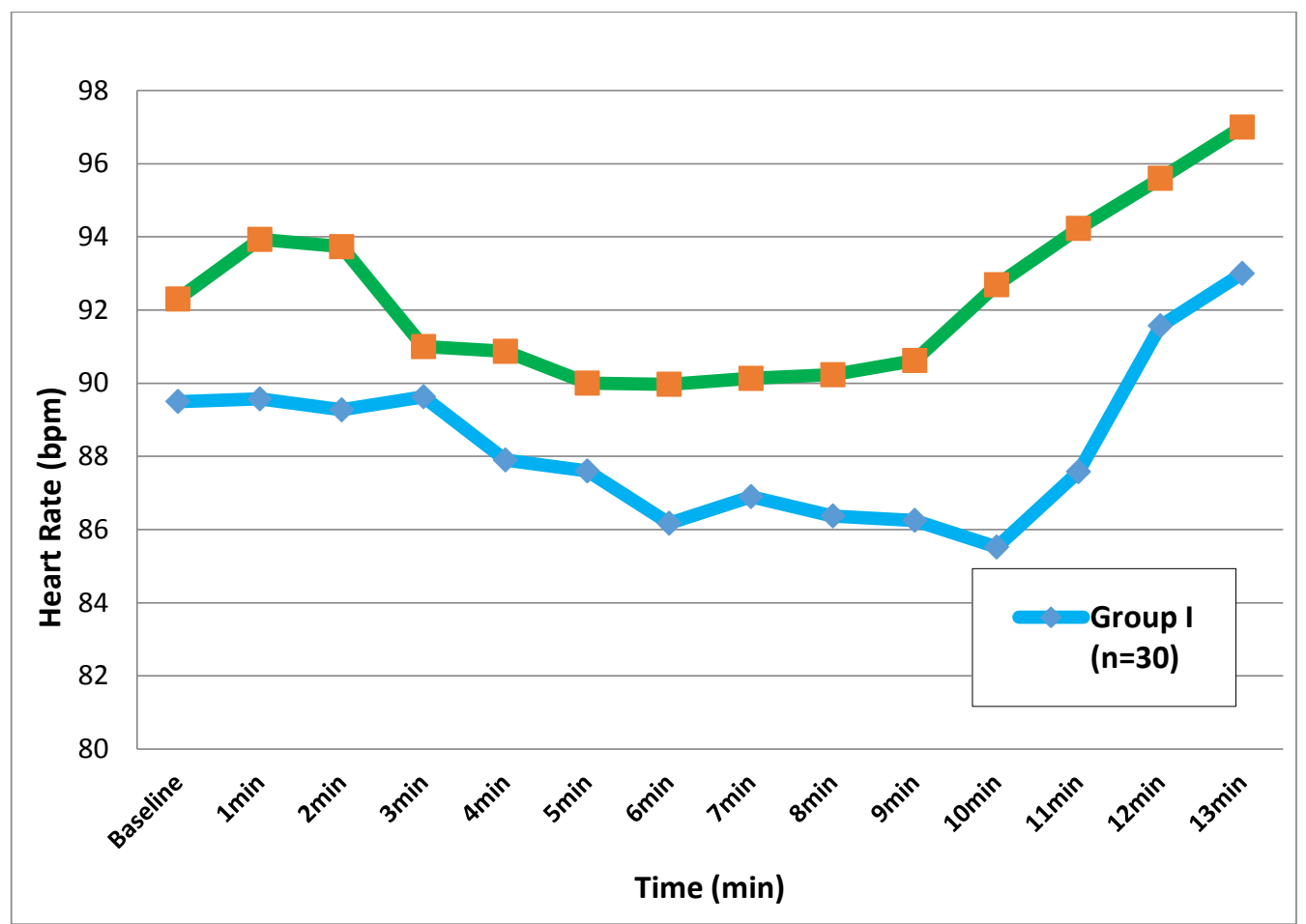

Fig 2: Comparison of heart rate among two groups 


\section{Discussion}

Caesarean section is one of the oldest operations in recorded history, anaesthesia for caesarean section is just a century old and is not bereft of controversies. The main drawback with this technique is the risk of hypotension especially in gravid parturients.

Dinesh Sahu et $\mathrm{al}^{9}$ found that maternal hypotension during spinal anaesthesia for caesarean delivery was a persistent problem in approximately $85 \%$ of cases. This is in spite of pregnant patients having $40-50 \%$ of more blood volume at term compared to non-pregnant patients. Pregnant patients at term are more prone to develop hypotension due to the occurrence of aortocaval compression by the fetal head and higher level of sympathetic blockade owing to increased spread of local anaesthetic in the cerebrospinal fluid. Hypotension, hazardous to both mother and the fetus, is better prevented than treated. Blood pressure is usually maintained in the face of vasodilation, caused due to factors other than central neural blockade, by a reflexive increase in cardiac output. However, in the presence of spinal induced venodilation, venous return is reduced to an extent that cardiac output cannot increase and is often reduced. The result is severe hypotension with reduced uteroplacental perfusion and APGAR score.

It has been shown that the percentage decrease in placental perfusion is related to the percentage reduction in maternal arterial pressure and not to the absolute reduction in pressure. For the purposes of this study, hypotension was defined as a decrease in arterial pressure greater than $20 \%$ from baseline systolic pressure. ${ }^{10}$

Most strategies for decreasing the incidence of hypotension during spinal anaesthesia for cesarean delivery have not proved to be reliable. Although early work suggested that intravenous prehydration with crystalloid solutions was effective, this has been questioned in more recent articles. Use of colloid solutions may be more effective than crystalloids but the benefits are still limited, and infusion of large volumes of colloid may have other risks, including fluid overload, interference with coagulation and anaphylactic reactions. Compression of the lower limbs has been described as an alternative technique but is not convenient and is not popular. Traditionally ephedrine has been recommended in this role. A number of other studies have recently reported on the use of $\alpha$ agonists in obstetrics. ${ }^{11}$

Pure $\alpha$-agonist vasopressors initially were considered contraindicated in obstetrics, because early experimental studies reported a substantial decrease in uteroplacental blood flow linked to their vasoconstrictive properties. However, doses used in these studies were much higher than those needed clinically in humans, although they were appropriate to the species studied to restore spinal anaesthesia induced hypotension. In addition, a more recent experimental study suggested that pregnancy is associated with an attenuated uterine vascular response to phenylephrine. Clinical studies in women undergoing scheduled cesarean delivery have confirmed that small $(40-100 \mu \mathrm{g})$ bolus doses of phenylephrine used to counteract hypotension during epidural or spinal anaesthesia were effective and as safe as ephedrine bolus doses for the mother and the neonate.

The usual approach to use vasopressors is reactive rather than proactive. Spinal anaesthesia induced maternal hypotension is allowed to develop and then treated accordingly. We have, instead, studied prophylactic phenylephrine infusion and compared it with control group which is not receiving prophylactic infusion for maintenance of maternal blood pressure during spinal anaesthesia in caesarean section and its effects on APGAR score and umbilical artery blood $\mathrm{pH}$.

\section{Heart rate:}

In this study phenylephrine infusion group had significant fall in heart rate when compared to control group. Heart rate increased more than basal valves in control group possibly due to beta agonistic action of ephedrine. Possible bradycardia associated with spinal anaesthesia may have been annulled by ephedrine due to 
increased chronotropic action by stimulation of beta receptors.

Our result correlates with the study by Dinesh Sahu et $\mathrm{al}^{9}$, in which the heart rate values significantly reduced immediately after bolus administration of phenylephrine where as in ephedrine group heart rate stayed higher till 30 minutes after bolus administration. Incidence of bradycardia was $5 \%$ of the phenylephrine group and none in ephedrine group.

A study done by Thomas D.G. et al ${ }^{12}$ showed $58 \%$ incidence of bradycardia after administration phenylephrine $100 \mathrm{mcg}$ IV bolus compared to the ephedrine group. These finding were consistent with our study and suggests that phenylephrine causes reflex bradycardia via baroreceptor mediated reflex mechanism, which was transient.

The above findings suggests phenylephrine infusion keeps the heart rate uniformly but on a lower side compared to the ephedrine boluses where a fluctuation in the heart rate was observed.

\section{Blood pressure:}

In our study, we also observed that the incidence of hypotension is $6.66 \%(n=2 / 30)$ in infusion group and $96.66 \%(n=29 / 30)$ in control group. This reflects a more stable management of blood pressure can be achieved by phenylephrine infusion as it uniformly maintains the plasma level of this vasopressor.

In our study we observed that the SAP was consistently higher in the infusion group when compared to the control group. This probably was due to the stimulation of post synaptic $\alpha$ receptors by phenylephrine resulting in intense arterial and peripheral venoconstriction causing rise in blood pressure.

This results correlates with the study done by Dinesh Sahu et $\mathrm{al}^{9}$, in their study SBP, DBP, MAP values in phenylephrine group was maintained higher than ephedrine immediately after bolus administrations.

Hiroshi Ueyama et $\mathrm{al}^{13}$ studied prophylactic infusion of ephedrine $40 \mathrm{mg}$, phenylephrine 250 mcg for 5minutes immediately after spinal anaesthesia, showed incidence of hypotension $10 \%$ in ephedrine group and none in phenylephrine group. They concluded that no incidence of hypotension in phenylephrine group was due to increase in total vascular resistance by alpha agonistic activity of the drug.

However, we found that the required dose of phenylephrine was higher. At term, uterine vascular bed is maximally vasodilated and unable to auto regulate when perfusion pressure is reduced. Consequently a higher adrenoceptor density renders uteroplacental blood flow potentially vulnerable to vasoconstriction induced by $\alpha$ adrenergic agonists. Indeed infusion of phenylephrine at rate of $8 \mathrm{mcg} \mathrm{kg}-1 \mathrm{~min}-1$ has been reported to decrease ovine uteroplacental blood flow by $50 \%$ however the relationship between phenylephrine dose and uterine vascular resistance is not linear and dramatic increases in uterine vascular resistance seen only to appear with doses greater than $100 \mathrm{mcg}$ min- 1 thus, the satisfactory fetal outcome in human studies may simply reflect the lower doses used. This which is consistent with our study where the infusion rate was $100 \mathrm{mcg} \min -1 .^{12}$

\section{Neonatal outcome}

The APGAR scores and measured umbilical artery blood $\mathrm{pH}$ were used to indicate adequacy of placental perfusion. Despite periods of maternal hypotension in control group and decreased heart rate in infusion group, we found no significant difference in either APGAR score or UAB $\mathrm{pH}$ between two groups. This could be due to immediate correction of hypotension episodes and thus maintenance of uteroplacental perfusion in both the groups.

The APGAR score were similar in the groups and never less than 8 , this result correlated well with studies done by Alahuhta et $\mathrm{al}^{14}$ with ephedrine $5 \mathrm{mg}$ or phenylephrine $100 \mathrm{mcg}$ followed by 50mg hr-1 ephedrine and 1000 mcg hr-1 phenylephrine, showed no change in APGAR scoring. 
Ramanathan et al ${ }^{15}$ studied $5 \mathrm{mg}$ ephedrine and phenylephrine $100 \mathrm{mcg}$ IV bolus, they concluded that transient maternal hypotension does not affect neonatal acid - base status, both ephedrine and phenylephrine do not cause fetal acidosis, when used for treating maternal hypotension.

Also, Hall and coworkers ${ }^{10}$ found that despite periods of maternal hypotension, which occurred longer than 2 minutes, and periods of maternal bradycardia, there was no significant difference in either APGAR scores or blood-gas data between the groups.

The incidence of arterial $\mathrm{pH}<7.18$ was greater than desired. However this is not new information. Several studies have reported a surprisingly high incidence of acidosis (not accompanied with neonatal depression) after spinal anaesthesia for cesarean deliveries. ${ }^{16}$ Another mechanism for decreased uterine artery blood $\mathrm{pH}$ is by prolonged period of decreased maternal cardiac output occurring before delivery. Robson and colleagues $^{17}$ found that UAB $\mathrm{pH}$ correlated well with maternal cardiac output (but not with BP itself).

It was also shown by Joupilla and colleagues ${ }^{18}$ that intravenous preload maintains placental blood flow despite moderate reduction in maternal pressure, thus minimizing fetal acidosis. In our study we had taken precautions by preloading the patient's with $10 \mathrm{ml} \mathrm{kg-1}$ of ringer lactate solution which probably would have maintained placental blood flow during hypotensive episodes lasting longer than 2 minutes.

In our study, the observed decrease in UAB $\mathrm{pH}$ without a reduction in APGAR scores may be due to reduced cardiac output during delivery and the maintenance of uterine perfusion by adequate preload.

\section{Non hemodynamic side effects:}

In our study group none of the patients who received phenylephrine had nausea or vomiting.This results correlates with the study done by Cooper and colleagues ${ }^{19}$ in which nausea and vomiting were less frequent with phenylephrine (nausea 17\% and vomiting 0\%). They suggest that the possible explanation for nausea and vomiting is an increase in vagal tone following reduction of preload. Cardiac preload decreased in spinal anaesthesia, but phenylephrine a pure $\alpha$ - agonist, provides better venoconstriction, reducing the decrease in cardiac preload and diminishing the vagal reflex which is the cause for lower incidence of nausea and vomiting.

\section{Conclusion}

Prophylactic phenylephrine infusion is an effective and simple method of reducing the incidence of hypotension during spinal anaesthesia for caesarean delivery, with no adverse effect on neonatal outcome.

\section{References}

1. Clyburn P. Spinal anaesthesia for caesarean section: time for re-appraisal (Editorial) Anaesthesia 2005; 60:633-635.

2. Riley ET, Cohen SE, Rubenstein AJ, Flanagan B. Prevention of hypotension after spinal anaesthesia for caesarean section: $6 \%$ hetastarch versus lactated Ringer's solution. Anaesth Analg 1995; 81:838-42.

3. Eloner H, Barcohana J, Bartosheck AK. Influence of postsponal hypotension on fetal electrogram. Am J Obstet Gynecol 1960; 80:560-572.

4. Corke BC, Datta S, Ostheinar GW et al. Spinal anaesthesia for caesarean section. The influence of hypotension on neonatal outcome. Anaesthesia 1982; 37:658-662.

5. Jackson R, Reid JA, Thorburn J. Volume preloading is not essential to prevent spinal induced hypotension at caesarean section. Br J Anaesth 1995; 75:262-5.

6. Karinen J, Rasonen J, Alahuhta S, Jouppila $\mathrm{R}$ and Jouppila P. Effect of crystalloid and colloid preloading on uteroplacental and maternal Hemodynamic state during spinal anaesthesia for caesarean section. $\mathrm{Br} \mathrm{J}$ Anaesth 1998.75:531-35. 
7. McCrae AF, Wild Smith JAW. Prevention and treatment of hypotension during central neural block. Br J Anaesth 1993; 70:672-680.

8. Ngan Kee WD, Khaw KS, Ng FF, Lee BB. Prophylactic phenylephrine infusion for preventing hypotension during spinal anaesthesia for caesarean delvery. Anesth Analg. 2004; 98:815-21.

9. Sahu D, Kothari D, Mehrotra A. Comparison of Bolus Phenylephrine, Ephedrine, and Mephentermine for maintenance of arterial pressure during spinal anesthesia in caesarean section - a clinical study. Indian J Anaesth 2003; 47(2):125-128.

10. Hall PA, Bennett A, Wilkes MP, Lewis M. Spinal anaesthesia for Caesarean section: comparison of infusions of phenylephrine and ephedrine. Br J Anaesth 1994; 73: 471-4.

11. Pinto V., Jaysundara N., Abeysundara A., Ekanayake S. Use of phenylephrine as vasopressor of choice to prevent hypotension following spinal anaesthesia in LSCS. SL J Anaesth 2009; 16(2):74-78.

12. Thomas DG, Robson SC, Redfern N, Hughes D, Boys RJ. Randomized trial of bolus phenylephrine or ephedrine for maintenance of arterial pressure. During spinal anaesthesia for caesarean section. $\mathrm{Br}$ J Anaesth 1996; 76:61-65.

13. Hiroshi U, Yuki H, Masaki T, Takashi M. Maternal Cardiovascular Effects of Prophylactic Ephedrine and Phenylephrine for Elective Cesarean Section Undergoing Spinal Anesthesia. Anesthesiology 2002; 96: A 1051.

14. Alahuhta S, Rasanem J, Joupilla P, Joupilla R, Hollmen AI. Ephedrine and phenylephrine for avoiding maternal hypotension due to spinal anesthesia for caesarean section. Int $\mathbf{J}$ ObstetAnaesth 1992; 1:129
15. Ramanathan S, Friedman S, Moss P, Arismendy J, Turndorf H. Phenylephrine for the treatment of maternal hypotension due to epidural anesthesia. Anaesth Analg 1984; 63:262.

16. Mercier JF, Riley ET, Fredericson WL, Roger-Christoph S, Benhamou D, Cohen SE. Phenylephrine added to prophylactic ephedrine infusion during spinal anesthesia for elective Cesarean section. Anesthesiology 2001; 95:668-74. 16(2): $74-78$.

17. Robson SC, Boys RJ, Rodeck C, Morgan $\mathrm{B}$ : Maternal and fetal haemodynamic effects of spinal and extradural anaesthesia for elective caesarean section. $\mathrm{Br} \mathrm{J}$ Anaesth 1992; 68:54-9.

18. Jouppila P, Jouppila R, Barinoll $\mathrm{T}$, Koivula A. Placental blood flow during Caesarean section performed under subarachnoid blockade. $\mathrm{Br} \mathrm{J}$ Anaesth 1984; 56:1379.

19. Cooper DW, Carpenter M, Mowbray P, Desira WR, Ryall DM, Kokri MS. Fetal and maternal effects of phenylephrine and ephedrine during spinal anesthesia for Cesarean delivery. Anesthesiology 2002; 97: 1582-90. 